were carried out with a hollow tin sphere of the same radius, the spherical space in the middle being equal in volume to one half the volume of the sphere. The magnetic moments produced in the hollow sphere were two to three times greater than those obtained with the solid sphere.

In all these experiments the magnetic field was produced by a cylindrical coil in the middle of which the sphere was placed, all iron being excluded. Although the field near the sphere was thus fairly homogeneous, we think it possible that the observed phenomena may be influenced by slight inhomogeneities of the external field. In a completely homogeneous field it would seem possible that the method of cooling might affect the results. In order to test this, we cooled the spheres from the poles and also from the equator. This did not seem to make any difference, the magnetic moment observed being of the same order of magnitude in either case.

As a result of these experiments, it seems certain that the effective permeability of substances when they become supraconducting decreases, as observed by Meissner and Ochsenfeld. On the other hand, it appears clear that under our experimental conditions the permeability does not vanish entirely, as might be expected in view of the almost infinite conductivity, or if it does vanish, it only does so in certain regions and not throughout the whole volume of the supraconductor.

In conclusion, we would like to express our thanks to $\mathrm{Mr}$. T. C. Keeley for his advice and assistance in various phases of the work.

K. Mendelssohn.

Clarendon Laboratory, J. D. BАввтTT.

Oxford.

Feb. 17.

' Comm. Leiden, Suppl., Nr. 50 b, 1924.

Naturwiss, 21, 787; 1933.

\section{Some Thermal Properties of Condensed Helium}

IN the following communication we give the results of some preliminary measurements with condensed helium :

1. The heat of fusion is $6 \cdot 75 \mathrm{cal} . / \mathrm{gm}$.-atom at $4 \cdot 0^{\circ}$ and $5 \cdot 1$ call at $3 \cdot 4^{\circ}$.

2. The density of solid helium in equilibrium with the liquid phase is 0.23 at $4 \cdot 0^{\circ}$ and 0.22 at $3 \cdot 6^{\circ}$.

3. The density and compressibility of liquid helium have been measured at $2 \cdot 4^{\circ}$ and $4 \cdot 5^{\circ}$. They are in good agreement with the data recently published by Keesom ${ }^{1}$. We would add only that the compressibility still falls appreciably with higher pressure.

4. The compressibility of solid helium could be measured roughly, the result being about $1.5 \times 10^{-3}$ reciprocal atm. at $3 \cdot 7^{\circ}$ and $115 \mathrm{~atm}$.

5 . The specific heat of solid helium was determined between $2 \cdot 7^{\circ}$ and $3 \cdot 7^{\circ}$ at a density of $0 \cdot 23$. $C_{v}$ agrees well in this region with a Debye function for $\Theta=32 \cdot 5^{\circ}$. This small value (the smallest hitherto observed), which we find in spite of the small atomic weight, is another consequence of the very weak interatomic forces. From $\Theta=32 \cdot 5^{\circ}$ follows a zero point energy of $73 \mathrm{cal} . / \mathrm{gm}$.-atom, compared with a thermal energy of only 1 cal. at $4^{\circ}$. We may mention that this value agrees well with the deviation from Trouton's rule if we attribute this to the zero point energy ${ }^{2}$.

6. Measurements of adiabatic expansion were carried out in connexion with a proposed procedure ${ }^{3}$ for lowering temperatures by changing the volume of condensed helium. The coefficient $\gamma=-(\delta \ln T / \delta \ln v)_{8}$ was measured for the liquid phase as a function of temperature and pressure. We may mention as an example, that starting at $4 \cdot 6^{\circ}$ and 130 atm., $2 \cdot 4^{\circ}$ is reached by expanding to the vapour pressure. Expansion experiments with solid helium have not yet been carried out, nor accurate experiments on the more easily made adiabatic expansion starting from the solid at equilibrium pressure. We have done only one preliminary experiment starting from partly solidified helium at $4^{\circ}$, and this showed an appreciable increase in the cooling effect as compared with the effect obtained with only liquid present. (The data given above, however, enable us to calculate these cooling effects. Starting with solidified helium at the equilibrium pressure at $4^{\circ}$, one should reach a temperature of $1 \cdot 4^{\circ}$ by expanding to the vapour pressure. As according to the measurements of Keesom ${ }^{4}$ on the thermal expansion an adiabatic expansion of the liquid below the $\lambda$-point should lead to a temperature rise, a lower temperature will be obtained by expanding only until the substance is just melted. Keesom's entropy diagram of the liquid shows that in this case a temperature $0.15^{\circ}$ lower should be reached. Definite predictions of the cooling effects when starting at lower temperatures cannot be given yet.)

7. The knowledge of the specific heat of the solid phase enables us to fix the zero point of entropy according to Nernst's Theorem. The absolute value of the entropy of the liquid can now be calculated using the values of the heat of fusion, the data of adiabatic expansion and the specific heats. Connexion can be made through the known values of vapour pressures and heats of evaporation with the theoretical value of the entropy of the gas, putting the statistical weight equal to unity according to the spectroscopic observations. We find good agreement, the resulting chemical constant being -0.62 compared with the theoretical value -0.68 .

The investigations were carried out in 1932 in Breslau. Their continuation is being undertaken in the Clarendon Laboratory.

Sofia.

R. KaIsChEW.

Oxford.

Feb. 25 .

1 W. H. and A. P. Keesom, Physica, 1, $128 ; 1933$.

${ }^{2}$ K. Bennewitz and F. Simon, Z. Phys., 16, 197 ; 1923.

${ }^{3}$ F. Simon, $Z$. Phys., 81, 824, 838; 1933 ; see also W. Meissner, Z. Phys., 81, 832 ; 1933; and W. H. and A. P. Keesom, Physica, 1 , $161 ; 1934$

W. H. and A. P. Keesom, Kon. Akad. v. Wetensch. Amsterdam, 36, Nr. 5; 1933 .

5 W. H. and A. P. Keesom, Physica, 1, 161; 1934.

\section{A Mercury-Sealed Water-Cooled Rotating X-Ray Target}

INSPIRED by the magnificent X-ray tube with spinning target designed by Müller ${ }^{1}$ and now in operation at the Davy Faraday Laboratory of the Royal Institution, we have considered the feasibility of completely sealing off the rotating anode by means of mercury after the manner of the classical Torricelli experiment.

At first sight such a proposition seems absurd for 